\title{
Control of Vector Borne Diseases of Public Health Importance: The Role of Integrated Vector Management and Indigenous Knowledge
}

\author{
Oda Gizaw $^{1} \quad$ Gelaye Gebisa $^{2} \quad$ Biruk Bogale $^{2} \quad$ Wondosen Zewdu $^{3} \quad$ Geda Regassa $^{4}$ \\ 1.Department of Animal Science, Mettu University, Ethiopia \\ 2.School of Animal and Range Sciences, Hawassa University, Ethiopia \\ 3. Gorche District Office of Livestock and Fisheries, Ethiopia \\ 4.Adaa District Office of Livestock and Fisheries, Ethiopia
}

\begin{abstract}
This study reviews about the role of indigenous knowledge and integrated vector management for controlling vector borne diseases of public health importance with the aim of providing summarized information to the beneficiaries. Now a day's vector-borne diseases are the major illnesses of humans and animals. Many of vectorborne pathogens affect humans are of zoonotic origin. Reservoir hosts of these pathogens include wildlife, livestock, and companion animals. An important vector borne diseases with zoonotic potentials includes Leishmania, Dengue, Nipah virus, Rift Valley Fever, African Trypanosomiasis, Chagas disease, Yellow Fever, Japanese Encephalitis, Onchocerciasis etc. These vector-borne diseases pose an increasingly wider threat to global public health, both in terms of the number of people affected and their geographical spread. For many vectorborne diseases, there are no vaccines, and their drug resistance characteristic is an increasing threat for public health. To achieve progress and stability in animal and human health, vector borne diseases have to be controlled effectively. In olden days, people traditionally practiced various indigenous knowledge to control insect and other vectors transmit diseases between animals and humans. Indigenous knowledge expresses traditional knowledge, rural knowledge as well as ethno science. Studies indicate that veterinary medicine, as practiced today, has its roots in herbal medicine, which has been practiced since ancient times. As many vector control methods are effective against multiple diseases, they can be integrated together to combat multiple diseases at once. Finally we recommend an Integrated Vector Management as the process for developing and implementing strategies of WHO for controlling vectors besides using an indigenous knowledge.
\end{abstract}

Keywords: Indigenous Knowledge, Integrated Vector Management, Vector-borne diseases

DOI: $10.7176 /$ JNSR/9-22-03

Publication date: November $30^{\text {th }} 2019$

\section{Introduction}

Bacterial, viral and parasitic diseases are vector-borne diseases which transmitted by insects such as mosquitoes, lice, sand flies and fleas called vectors [1,2]. Now a day, vector borne diseases are the major illnesses of humans and animals caused by pathogens and parasites. Every year more than one billion vector-borne diseases develop globally with a cause for the fatality of one million lives [3, 4, 5]. Universally an important vector borne diseases with zoonotic potentials included Leishmaniasis, Dengue, Nipah Virus, Elephantiasis, Schistosomiasis, Trypanosomiasis, Chagas disease, Yellow Fever, Japanese Encephalitis, Onchocerciasis etc. [2].

Indigenous knowledge expresses the traditional and rural knowledge as well as ethno science and it evolved through many years of regular experimentation on the day-to-day life and available resources surrounded by the community. Study by Matsika [6] indicated that, traditional knowledge has been integrated with agriculture activities of the population like, animal health. The study reported by Teklay [7] indicated that majority of the Ethiopian livestock owners mainly depend on traditional healers and herbalists due to inadequate modern veterinary services in Ethiopia and its inaccessibility in most parts of the country. Traditional veterinary practices include mechanical, physical, pharmacological, surgical, rituals and managerial methods of treatment using faunal medicine [8].

Integrated vector management (IVM) is a rational decision-making process to optimize the use of resources for vector control and it requires a management approach that improves the efficacy, cost effectiveness, ecological soundness and sustainability of vector control interventions with the available tools and resources [3]. This management involves the use of a range of proven vector control methods either alone or in combination.

In general, traditionally regarded as a problem for countries in tropical settings, vector-borne diseases pose an increasingly wider threat to global public health, both in terms of the number of people affected and their geographical spread [9]. According to this report of WHO [9] one sixth of the illness and disability suffered worldwide is due to vector-borne diseases, with more than half the world's population currently estimated to be at risk of these diseases. The poorest segments of society and least-developed countries are the most affected. Vectorborne diseases affect urban, peri-urban and rural communities but thrive predominantly among communities with 
poor living conditions particularly lack of access to adequate housing, safe drinking water and sanitation. Malnourished people and those with weakened immunity are especially vulnerable [9]. Illness and disability prevent people from working and supporting themselves and their family, causing further hardship and impeding economic development. For many vector-borne diseases, there are no vaccines, and drug resistance is an increasing threat. Control vector-borne diseases therefore play a central role in poverty reduction and economic development [10]. Vector control plays a vital role and is often the only way to prevent disease outbreaks. Therefore, the objectives of this review was to compile an information about the role of indigenous knowledge and integrated vector management for controlling vector borne diseases of public health importance.

\section{An overview on vector borne diseases}

Vector-borne diseases are the major illnesses of man and animals now a day and caused by pathogens and parasites. The entry and development of an infectious agent take place in the body of a person or animal [1,2,11]. Vectorborne diseases account for about $17 \%$ of an estimated global burden of infectious diseases and exert an enormous toll on the continent of Africa. They result in loss of productivity, school absenteeism, and aggravation of poverty, high costs for health care and burden on public health services $[1,12]$.

In ancient periods, insects were very important in the transmission of communicable diseases and it was mostly related with the definition of vector. Later on the term vector has been used more widely to include other non-human animals including snails, dogs and rats [4]. Therefore alternative definitions are found for vectors and it can be defined as arthropods and other invertebrates which transmit infection by inoculation into or through the skin or mucous membrane by biting or by deposit of infective materials on the skin or on food or other objects $[4,10,13,14]$. Important vectors transmitting zoonotic diseases are mosquitoes, flies, fleas, lice, biting flies, bugs, ticks, mites, snails, etc. which actively transmit pathogens (Table 1) from an infected reservoir host animal to another individual. Many of these are blood sucker, which ingest disease-producing microorganisms during a blood meal from an infected host (human or animal) and later inject it into a new host during their subsequent blood meal. Mosquitoes are the best known vector for transmission of a number of diseases [5, 15, 16, and 18]. The chain of infection for insect borne diseases involves a pathogenic organism in an infected person or animal, an arthropod to transmit the disease and a susceptible human or animal.

Vector borne diseases are transmitted both in a mechanical and biological ways. In the first way of transmission, the diseases causing agents are transported by flying insects (without any development in the vector) through contaminated legs, wings, mouth parts or through excretory materials and in biological way of transmission, the disease causing agents complete its development cycle in the vectors before transmit in to the vertebrate hosts $[1,2,14]$. Many vector-borne pathogens affecting humans are of zoonotic origin. Reservoir hosts of these pathogens include wildlife, livestock, and companion animals [10]. Many of these diseases of humans and animals are increasing in terms of incidence and geographical distribution over the world. The existing and changing distribution of vector-borne pathogens directly affects the daily routine of physicians and veterinarians $[5,19]$. Indeed, the introduction and establishment of pathogens into new areas may impact on their clinical practice, not only because they may be unprepared for diagnosing new diseases, but also because co-infections may mask the clinical picture and delay the final diagnosis, eventually increasing the risk of life-threatening complications $[5,10,12]$. The transmission mechanism of infectious agents such as virus through which pathogen is spread from a reservoir to human being is very significant. Usually each type of infectious agent is ordinarily spread by only one or a few of the different mechanisms. The significance of vector efficiency in disease transmission from reservoir to host is related to many factors. Some of the factors are species-related such as vector reproductive capacity, physiology, morphology and genetics [15]. Moreover, some other factors that affect the vectors ability to transmit disease are physical and related to environmental conditions, such as temperature, moisture, rainfall, weather, geographical location and wind. Peoples living in a field environment must break the chain of infection for insect borne diseases or arthropod injury by limiting arthropod vector exposures [5, 19].

Risk factors associated with vector-borne zoonoses are: globalization of trade and travel, unplanned urbanization and environmental devastation due to climate change poses significant impact on vector borne disease transmission in recent years; due to lack of awareness of some vector borne diseases like dengue, chikungunya, Nipah and West Nile virus etc. have emerged in countries where they were previously unknown; changes in agricultural practices, changes in the breeding places of vectors due to deforestation, draughts, variation in temperature and rainfall affect increase transmission or wipe out few of the vector borne diseases; lack of proper education, improve awareness and monitoring about the distribution of vectors, vector-borne diseases and other climate-sensitive diseases; a crucial element in catching vector-borne diseases is the behavioral change of people during travel and tourism and searching for feed and living places in wild environment; poor national economy and lack of mobilizing fund for developing technical expertise and lack of draconian measures often emerge or reemerge VBDs in developing worlds [5]. 
Table 1: Important vectors and disease transmission mechanisms

\begin{tabular}{lll}
\hline Vector & Diseases & Mechanism \\
\hline Housefly & Diarrhoeal diseases, worms, food poisoning, infective hepatitis & Mechanical \\
Mosquito & Malaria, yellow fever, filariasis, dengue fever, Rift Valley Fever & Biological \\
Louse & Typhus fever, relapsing fever, dermatitis & Biological \\
Mite & Scabies, chigger & Biological \\
Flea & Plague, murine typhus/endemic typhus & Biological \\
Sandfly & Leishmaniasis & Biological \\
Blackfly & Onchocerciasis & Biological \\
Bedbug & Dermatitis, Chagas disease & Biological \\
Tsetse fly & Sleeping sickness (trypanosomiasis) & Biological \\
Freshwater snail & Schistosomiasis & Biological \\
Dog & Rabies & Biological \\
\hline
\end{tabular}

Source : (Khan, 2015)

Furthermore, descriptions about some diseases transmitted by vectors are indicated as follows;

\section{Rift Valley Fever (RVF)}

Rift Valley Fever is arthropod-borne viral haemorrhagic zoonoses that cause disease in both animals and humans leading to high morbidity and mortality. It is biologically transmitted by mosquitoes of the genera Aedes that breed in low depressions which flood after heavy rainfall. It is endemic in sub-Saharan Africa because of transovarian transmission in Aedes mosquitoes. The disease affects mainly cattle, sheep, goats, camels and buffaloes during climatic conditions favouring hatching of virus infected eggs of flood water mosquitoes; the Aedes from their endemic sites $[2,5,9]$. This disease causes heavy economic losses and disrupts livelihoods of livestock producers through abortions, animal deaths, restrictions of trade which may last several years through quarantines, consumer boycotts of meat and costs of controlling the disease [20]. It has also a public health importance, RVF virus is thought to be arthropod vector based and propagated during epizootics or in endemic regions [5]. Human's also has an exposure to be infected with the virus related to the occupational including in laboratory workers, through intensive contacts with and handling of infected livestock (housing animals indoors with household members or their products), by aerosols of blood or amniotic fluids during parturition, slaughter, autopsy, bleeding, evisceration, preparation of food, processing tissues, and hides and skins or hand milking and consumption of raw milk. It may also be transmitted through inoculation via a cracked skin. In humans, the disease manifests itself as retinitis, meningoencephalitis and haemorrhagic fever [21].

\section{Leishmaniasis}

Leishmaniasis (dom dom fever) are protozoan parasites of great medical and veterinary significance and transmitted to a susceptible host by infected female phlebotomine sand flies [2]. Out of 30 reported species of leishmania, over 20 of them can infect humans and is transmitted to humans by more than 30 different species of phlebotomine sandflies. Leishmaniasis in human consists of four main clinical syndromes. Those syndromes are Cutaneous Leishmaniasis (most common), Muco-Cutaneous Leishmaniasis (also known as espundia), Visceral Leishmaniasis (VL, popularly known as Kala-azar, most serious form) and post-kala-azar dermal leishmaniasis (PKDL, chronic condition) [5]. Leishmania donovani and Leishmanial infantum are the two types of leishmanial species which are cause for Visceral Leishmaniasis depending on the geographical area. Leishmania infantum infects mostly children and immunosuppressed individuals, whereas Leishmanial donovani infects all age groups. Visceral Leishmaniasis is among the most neglected diseases based on dearth of research and notable lack of innovations in disease treatment and control. It is by far the most devastating form of Leishmaniasis, and is nearly $100 \%$ fatal if not treated. Natural vertebrate hosts of Leishmania parasites are humans, armadillos, sloths, dogs, cats, hyraxes, rats, gerbils, monkeys, opossums, horses, foxes, golden jackals etc. [1, 9, 15].

\section{Dengue Fever}

Dengue is the most rapidly spreading mosquito-borne viral disease in the world. Unlike other mosquitoes, Aedes aegypti, the main vector for dengue bites during the day. Aedes albopictus, a secondary dengue vector, can survive in cooler temperate regions $[5,9,10]$. There are four closely related serotypes of the virus that cause Dengue and the lifelong immunity developed after infection. In the past 50 years, incidence of dengue has increased thirty-fold with increasing geographical expansion to new countries and, in the present decade, from urban to rural settings. More than 2.5 billion people in the world are now at risk from dengue. Severe dengue also known as dengue haemorrhagic fever is found in tropical and sub-tropical locations in most Asian and Latin American countries. An estimated 500000 people with severe dengue require hospitalization each year, a large proportion of these are children. About $2.5 \%$ of those affected exposed for the death. There is no vaccine or any specific medicine to treat dengue $[2,9,1322]$. 


\section{Sleeping Sickness}

African Trypanosomosis (sleeping sickness) is an illness endemic to sub-Saharan Africa and limited to the continent of Africa where its insect vector, the tsetse fly, is found [23]. It is caused by two subspecies of the flagellate protozoan Trypanosoma brucei. There are two forms of sleeping sickness. The chronic gambiense form is found in Central and West Africa and although an animal can be found infected, the disease is maintained by transmission between the insect vector and humans. However, the animal reservoir is important in the acute rhodesiense form found in Eastern and Southern Africa [24]. The causal agent, Trypanosoma brucei rhodesiense, infects humans, wild animals and domestic livestock, which maintain infection between epidemics, and coexists in animals with a complex of pathogenic trypanosomes (T. congolense, T. vivax and T. b. brucei) that present a major problem for livestock keepers in Africa [25]. If not treated, the disease is always fatal in humans and treatment is expensive, normally costs from US\$150 to US\$800 per person. In the later stages of the disease treatment, it involves about 5\% mortality. Control is via the vector or the disease's human and livestock reservoirs $[1,9]$. For rhodesiense, the key to preventing the disease in people is now thought to be by treating the cattle reservoir, using drugs which are effective not only against the trypanosomes pathogenic to humans but also those which cause substantial losses to livestock production [24].

\section{Indigenous knowledge in the control of vector borne diseases}

Indigenous knowledge is a complex body of knowledge, skills and technology, which belongs to a particular geographical community. It expresses traditional knowledge, rural knowledge as well as ethno science. It evolved through many years of regular experimentation on the day-to-day life and available resources surrounded by the community. This knowledge has been integrated with agriculture activities of the population like, animal health [6]. Furthermore, indigenous knowledge has been recognized as a valuable input into modern industries such as pharmaceuticals, botanical medicines, cosmetics and toiletries, agriculture and biological pesticides. Most industries look for the time tested traditional knowledge information for developing novel products having commercial acceptability [26, 27]. Indigenous knowledge has been based on practical experience; it can be preserved and harnessed for the benefit of both present and future generations, belonging to humanity in general and its communities in particular [28, 29].

The study by Risiro [29] pointed about the introduction of western education and missionary activities in Africa watered down the value and respect given to indigenous knowledge and cultural beliefs. Some of this knowledge has been regarded as primitive and superstitious even though indigenous knowledge had been respected and practiced by indigenous people on their own custodians and legislators of environmental management. Furthermore the finding of Risiro [29] indicates that, an indigenous knowledge had ensured forests, water resources and animals protected from destruction and extinction. Different studies indicates that veterinary medicine which we practiced or used today has its roots in herbal medicine and which has been practiced since ancient times in China, India and the Middle East Asia. Middle East Asia is the center of veterinary and other medical knowledge in the early middle ages. With the spread of Islam, some of this knowledge made its way into Africa and adopted by stock raisers. Ethno veterinary medicine has been practiced as early as 1800 B.C. At the time of King Hammurabi of Babylon formulated laws on veterinary fees and charged for treating cattle and donkeys. Traditional veterinary practices have been around for a long time and remained the only medicine available until nineteenth century $[25,30]$. There are mentions of ethno veterinary medicines in the epic Mahabharata where the two Pandav brothers of Nakul and Sahadev were famed for their knowledge on treatment of horses and elephants, later there are reports where King Ashoka and Kanishka had established veterinary hospitals in their kingdom besides Sher Shah Suri had also established livestock husbandry department in his kingdom [8]. In China, the ancient philosophers Confucius and Shi Tzu had reported the importance of livestock, their husbandry, treatment, and its relationship with the prosperity of the agrarian community. In the same way over the centuries of Africa stockowners, through their own methods of trial and error have learnt a great deal about animal diseases and their treatments. These remedies are now widely used in light of the different Ethno veterinary practices in Africa. So far, several studies on Ethno veterinary medicine (EVM) have been conducted in Africa. In most sub-Saharan African countries, more than $80 \%$ of the population uses the services of traditional healthcare practitioners in combination with conventional healthcare systems $[8,30]$.

Ticks and tick borne diseases as well as, tsetse and Trypanosomosis, Rift Valley fever, etc. are major parasitic vector borne disease complexes with very serious effects on livestock production and productivity in the sub Saharan Africa. For many years, these diseases have been causing serious problems in livestock production especially among the poor rural farming communities. This problem is exacerbated by the farmers' lack of access to the conventional livestock management skills and financial resources to afford vaccines and curative substances. However, the stockowners use herbals with combination of other modern medicine to control tick invasion in their stock $[15,31,32]$.

Vector borne diseases have to be controlled effectively for achieving progresses and stability in animal and human health. In ancient days, people traditionally practiced various tips to control mosquitoes. They have been 
grouped into two main headings: Lifestyle oriented practices and Agriculture oriented practices [33, 19].

\section{Lifestyle oriented practices}

Based on the lifestyle oriented practices, human beings used to have various measures to control the menace of vectors. Those are described as follows.

Fogging techniques: As part of traditional practices in order to avoid mosquitoes and other harmful vectors, people have been used fogging techniques since early times. For fogging people used various techniques like burning cattle dung continuously around their housing, special combinations by indigenous experts (timbo, kobo, garlic), by burning dried leaves and plant parts and incensing by using cedar (Ejersa, Debeka, Muka-Arba, Chieka, etc.). Burning of these materials give some sort of fragrance to the surroundings on one hand and getting relief from flies, mosquitoes and other insect biting, and also believed to have religious and social importance [34].

Use of domestic plants of penetrating aromatic smell : Every house rears plants which produce aromatic penetrating smell e.g. Timbo, Kusaye, Asengira, Nini-zaf, Tenadam, Nech-sar, etc. which repel mosquitoes and other flies [7].

Oil bathing: To avoid mosquito and other insect bites people usually practice oil bathing (like applying fresh butter on uncovered body parts, rubbing oil on the body, etc.). It helps not only mosquitoes and flies avoidance but also has health significance [16].

Periodic decanting of waste water from containers: In order to collect water for livestock or for some other purposes, people collect water in vessels and sometimes thrown vessels contain waste water found almost in all houses. In such vessels mosquitoes used to lay eggs. To avoid mosquito breeding, people often decant water from the vessels regularly or avoid the vessels. This practice found to be a precautionary measure to control mosquitoes [17].

\section{Agriculture oriented practices}

According to the study indicated by Gubler [33], Agricultural oriented practices also helped human beings to control vectors. Agricultural oriented practices are described as follows.

Biological control : By rearing chicken, cat, etc and allow them to move freely around the house, people traditionally drive away insects (cockroaches, ticks, snails, worms, etc) and rodents those carry fleas, louse, and other harmful insects from human surroundings [35].

Burning of agricultural wastes: For achieving soil fertility, farmers often burn agricultural waste materials and its ash makes soil fertile on one hand and its fog repel mosquitoes from human beings on the other hand and also ticks dropped from animals for reproduction and their hatched eggs damage there [36].

Ploughing the farms and garden: Agricultural practices itself become a measure to avoid or reduce vectors menace. Farmers ploughed their farms and gardens to plant different varieties of plants in their agricultural fields and this process helps in avoiding of vectors like ticks, flies, mosquitoes, etc. by either burying them or distorting their environment [8].

Periodical cleaning for agriculture: For agriculture, farmers used periodic cleaning of bushy areas, where mosquitoes breed and rear. It is followed by filling up of ditches containing water. All these measures avoid mosquito's breeding surroundings [18]. Indigenous or traditional practices are getting much important which were ignored or lost due to the influence of modern technological approaches [28].

\section{Integrated Vector Management in the control of vector borne diseases}

Integrated vector management (IVM) is a rational decision-making process to optimize the use of resources for vector control. It requires a management approach that improves the efficacy, cost effectiveness, ecological soundness and sustainability of vector control interventions with the available tools and resources. Integrated approach is vital in successfully combating vector-borne diseases. Various key elements of IVM are Advocacy, social mobilization, strengthening of regulatory and legislative controls for public health, empowerment of communities, collaboration within health and other sectors in planning and decision-making, use of available resources for vector control, implementation of evidence-based strategies and capacity-building [23]. Though different integrals of IVM have been under implementation in isolation or in combination in different situation, there is a felt need that a comprehensive document describing IVM concept, its components and strategy for different diseases are compiled. Integrated control is another magical approach thought to solve all the difficulties encountered by vector control. In fact it is a philosophy and not a method and its efficacy will depend on the methods and tools which could be integrated $[1,3,13]$.

Integrated vector management (IVM) is the strategic approach to vector control promoted by WHO and it considers five key elements in the management process, namely: advocacy, social mobilization and legislation; the promotion of these principles in development policies of all relevant agencies, organizations and civil society; the establishment or strengthening of regulatory and legislative controls for public health; and the empowerment of communities. Collaboration within the health sector and with other sectors; the consideration of all options for 
collaboration within and between public and private sectors; planning and decision-making delegated to the lowest possible administrative level; and strengthening communication among policy-makers, managers of programmes for the control of vector-borne diseases, and other key partners. Integrated approach to disease control; ensuring the rational use of available resources through the application of a multi-disease control approach; integration of non-chemical and chemical vector control methods; and integration with other disease control measures. Evidencebased decision-making; adaptation of strategies and interventions to local vector ecology, epidemiology and resources, guided by operational research and subject to routine monitoring and evaluation. Capacity-building; the development of essential infrastructure, financial resources and adequate human resources at national and local levels to manage IVM programmes, based on a situation analysis [3, 23]

Integrated vector management (IVM) involves the use of a range of proven vector control methods, either alone or in combination; several methods may be used against a single disease, or a single or several methods may be used against several diseases. The vector control tools may be chemical or non-chemical. IVM can also supplement vaccines, mass drug administration or diagnosis and treatment for integrated disease control. In certain situations, IVM can be used for several diseases concurrently, because some vectors can transmit more than one human pathogen (e.g. Anopheles gambiae is involved in malaria as well as lymphatic filariasis transmission) and some interventions are effective against several vectors (e.g. long-lasting insecticidal nets are effective against malaria, lymphatic filariasis and leishmaniasis vectors) [23]. As many vector control methods are effective against multiple diseases, they can be integrated together to combat multiple diseases at once. The World Health Organization recommends "Integrated Vector Management" as the process for developing and implementing strategies for vector control. IVM focuses on utilizing preventative methods to control or eliminate vector populations $[9,15,10]$. Common vector control methods according to IVM measures are as follows:

Habitat and environmental control: Removing or reducing areas where vectors can easily breed can help limit their growth. For example, stagnant water removal, destruction of old tires and cans which serve as mosquito breeding environments, and good management of used water can reduce areas of excessive vector incidence. Further examples for environmental control are by reducing the prevalence of open defecation or improving the designs and maintenance of pit latrines. This can reduce the incidence offlies acting as vectors to spread diseases via their contact with feces of infected people [3]

Reducing contact: Limiting exposure to insects or animals that are known disease vectors can reduce infection risks significantly. For example, bed nets, window screens on homes, or protective clothing can help reduce the likelihood of contact with vectors. To be effective this requires education and promotion of methods among the population to raise the awareness of vector threats [3].

Chemical control: Insecticides, larvicides, rodenticides, lethal ovitraps and repellents can be used to control vectors. For example, larvicides can be used in mosquito breeding zones; insecticides can be applied to house walls or bed nets, and use of personal repellents can reduce incidence of insect bites and thus infection. The use of pesticides for vector control is promoted by the World Health Organization (WHO) and has proven to be highly effective [1].

Biological control: The use of natural vector predators, such as bacterial toxins or botanical compounds, can help control vector populations. Using fish that eat mosquito larvae or reducing breeding rates by introducing sterilized male tsetse flies have been shown to control vector populations and reduce infection risks [15].

\section{Conclusion and Recommendation}

Vector-borne diseases pose an increasingly wider threat globally both on animal and public health and also aggravate poverty. Since there are no directions or promotions to prevent many of vector-borne diseases, and drug resistance is an increasing threat. An effective control method to control vector-borne diseases are mandatory to achieve stability and progress in both animal and human health. Different control techniques has been using to tackle vector-borne diseases and the techniques are either the indigenous knowledge of the community which is made in traditional way or integrated vector management that developed by world health organization, or both. In early ages, people use their indigenous knowledge to control vectors transmitting diseases between animal and human and now days in most part of the world, especially in African, Asian and Latin American countries, and people use both the traditional knowledge and modern scientific methods. Complementing the modern vector control methods, like chemical using, with other effective control measures is currently a preferable system and this idea brought the process for developing and implementing strategies for vector control called integrated vector management which is the strategic approach to vector control promoted by WHO. Integrated vector management involves the use of a range of proven vector control methods, either alone or in combination. Finally, based on the above review, the following recommendations are strictly forwarded:

- Indigenous knowledge and traditional healers concerning vector-borne diseases should get the necessary recognition and protection by the educated community and local governments.

- Since indigenous knowledge about the control methods of vector-borne diseases of public health importance is organic, the veterinarian and public health professionals should adapt themselves to these practices to 
protect the ecology.

- Keeping the records of indigenous knowledge on the control of vector-borne diseases of public health importance and conducting researches to improve their efficacy is mandatory.

- The strategy of IVM should be developed and implement in complement with indigenous knowledge to protect animals and human from zoonotic VBDs.

- National and local governments should give serious attention on collaboration with WHO in control of VBDs through the strategy and process of IVM.

- For protection of animals and humans through provoking their immunity, there should be due attention on development and production of vaccines for VBDs.

- There should be development of strategies and draconian measures concerned about drug resistance threats on VBDs to overcome the challenges, the veterinarian, clinicians and public health professionals should play important parts in this area.

- Countries with high incidence and risk of VBDs, like Ethiopia, should develop national policy and strategic plan for IVM, national IVM implementation plan and national guidelines for IVM

- Awareness creation about vector-borne diseases, their public health importance and proper control methods should be made, especially, for people at higher risk

\section{References}

1. Chanda E., Govere M. J., Macdonald B. M., Lako L. R., Haque U., Baba P. S., Mnzava A. (2013): Integrated vector management: A critical strategy for combating vectorborne diseases in South Sudan. Malaria Journal 12: $1-9$.

2. Khan M. A. H. N. A. (2015): Important vector-borne diseases with their zoonotic potential: present situation and future perspective . Bangl J Vet Med 13: 1-14.

3. WHO (2012): Handbook for integrated vector management. France.

4. Institute of Medicine (2008): Vector-Borne Diseases: Understanding the Environmental, Human Health, and Ecological Connections. In: Washington, DC.: The National Academies Press.

5. Sarwar M. (2015): Insect Borne Diseases Transmitted by Some Important Vectors of Class Insecta Hurtling Public Health. International Journal of Bioinformatics and Biomedical Engineering 1: 311-317.

6. Matsika, C. (2012): Traditional African Education: Its Significance to Current Education Practices with Special Reference to Zimbabwe [dissertation]. Gweru, Zimbabwe: Mambo Press: 263 p.

7. Teklay A. (2015): Traditional medicinal plants for ethnoveterinary medicine used in Kilte Awulaelo District, Tigray Region, Northern Ethiopia. Advancement in Medicinal Plant Research 3: 137-150.

8. Lans C., Khan E. T., Curran M. M., McCorkle M. c. (2007): Ethnoveterinary Medicine: Potential Solutions for Large-Scale Problems. In. pp. 17-31.

9. WHO (2014): A global brief on vector-borne diseases.

10. Upadhyay A. K., Maansi, Parmar T., Singh P., Pathak A. P. (2018): Vector-Borne Zoonotic Diseases. J Anim Health Behav Sci 2: 1-6.

11. Sharma A., Gupta V., Khandelwal A. (2017): The knowledge, attitude and practices regarding commonly occurring mosquito borne diseases among people in catering area of urban health and training centre . International Journal of Community Medicine and Public Health 4: 2864-2869.

12. WHO (2017): Integrated vector management strategic framework for the Eastern Mediterranean Region 2016-2020. Cairo: CC BY-NC-SA 3.0 IGO.

13. Braks M., Giessen J., Kretzschmar M., Pelt W., Scholte E., Reusken C., Zeller H., Bortel W., Sprong H. (2011): Towards an integrated approach in surveillance of vector-borne diseases in Europe. Parasites \& Vectors 4: 1-11.

14. Kilpatrick A. M., Randolph S. E. (2012): Drivers, dynamics, and control of emerging vector-borne zoonotic diseases. Lancet 380: 1946-1955.

15. Benelli G., Jeffries L. C., Walker T. (2016): Biological Control of Mosquito Vectors: Past, Present, and Future. insects 7: 1-18.

16. Alobuia W. M., Missikpode C., Aung M., Jolly P. E. (2015): Knowledge, Attitude, and Practices Regarding Vector-borne Diseases in Western Jamaica. Annals of Global Health 81: 654-633.

17. Jaleel W., Saeed S., Naqash M. N., Saeed Q., Iqbal N. (2015): Indigenous knowledge about mosquito and its management in Punjab, Pakistan . Türk Tarým ve Doða Bilimleri Dergisi 2: 1-9.

18. Liu X., Wan F., Cirendunzhu, Cirenwangla, Bai Li., Pengcuociren, Zhou L., Baimaciwang, Guo Y., Dazhen, Xu J., Sang S., Li X., Gu S., Wu H., Wang J., Dawa, Xiraoruodeng, Liu Q. (2014): Community Knowledge and Experience of Mosquitoes and Personal Prevention and Control Practices in Lhasa, Tibet . Int J Environ Res Public Health 11: 9919-9937.

19. Hurwitz I., Fieck A., Read A., Hillesland H., Klein N., Kang A., Durvasula R. (2011): Paratransgenic Control 
of Vector Borne Diseases . Int J Biol Sci 7: 1334-1344.

20. Townson H., Natan MB., Zaim M., Guillet P., Manga L., Bos R., Kindhauser M. (2005): Exploiting the potential of vector control for disease prevention. Bulletin of the World Health Organization 83: 942-947.

21. Kioko, P. M. (2012): Food safety knowledge and practices among actors in beef chain with reference to rift valley fever outbreak in maragua district, kenya. MSc thesis, school of public health, Kenyatta university, Kenya.

22. Mukhtar M. (2013): Integrated Vector Management (IVM): Best way forward to control dengue in Pakistan. Bayer Public Health Journal 24: 1-7.

23. WHO (2016): A toolkit for integrated vector management in sub-Saharan Africa.

24. Giordani F., Mwenechanya R., Barret R. (2014): Advances in Understanding and Treatment of Human African Trypanosomiasis: Divergent Diseases Caused by Distinct Parasites. In: Handbook of Pharmacogenomics and Stratified Medicine . pp. 901-917.

25. Marandure T. (2018): Concepts and key issues of ethnoveterinary medicine in Africa: A review of its application in Zimbabwe . African Journal of Agricultural Research 11: 1836-1841.

26. Madhu S. T., Sirisha T. (2016): Indigenous Knowledge and Conservation of resources: A Case Study of Kurichchan of Ispathapalli Settlement at Krishnagiri District in Tamil Nadu . International Journal for Research \& Development in Technology 5: 64-69.

27. Temba S., Erasto P., Chacha M. (2018): Utilization of African ethnoveterinary information for management of livestock diseases. RUFORUM Working Document Series 14: 839-844.

28. Yongabi K. A., DeLuka L., Mshigeni K., Mwendwa S. K. K., Dudley A., Njuakom F. N. (2015): Can we exploit and adapt indigenous knowledge and ethno-botanicals for a healthy living in the face of emerging diseases like Ebola in Africa. American Journal of Clinical and Experimental Medicine Special Issue: Clinical Innovations, Developments in the Diagnosis, Managementand Prevention of Ebola Disease (Marburg fever) and Hemorrhagic Fevers 3: 24-28.

29. Risiro J. (2013): Indigenous Knowledge Systems and Environmental Management: A Case - Study of Zaka District, Masvingo Province, Zimbabwe. International Journal of Academic Research in Progressive Education andDevelopment 2: 1-8.

30. Wanzala W. (2017): Potential of Traditional Knowledge of Plants in the Management of Arthropods in Livestock Industry with Focus on (Acari) Ticks. Hindawi Evidence-Based Complementary and Alternative Medicine 1-33.

31. Dantas-Torres F., Chomel B. B., Otranto D. (2012): Ticks and tick-borne diseases: a One Health perspective. Trends in Parasitology 28: 437-446.

32. Endalachew B. E. (2015): Practices of ethnoveterinary against animal trypanosomosis in Southern Ethiopia . Global Journal of Biological and Biomedical Research 3: 132-136.

33. Gubler J. D. (1998): Resurgent Vector-Borne Diseases as a Global Health Problem. Emerging Infectious Diseases 4: 442-450.

34. Abera B. (2014): Medicinal plants used in traditional medicine by Oromo people, Ghimbi District, Southwest Ethiopia. Journal of Ethnobiology and Ethnomedicine 10: 1-15.

35. Grade J. T., Tabuti R. S. J., Van Damme P. (2009): Ethnoveterinary knowledge in pastoral Karamoja, Uganda. Journal of Ethnopharmacology 122: 273-293.

36. Melaku A. (2013): Ethnoveterinary Practices and Potential Herbal Materials for the Treatment of Ticks in North Gondar. Global Veterinaria 11: 186-190. 